\title{
Cooperative Relaying Scheme for Orthogonal Frequency and Code Division Multiple Access Uplink System
}

\author{
Jung-In Baik · Hyoung-Kyu Song
}

Published online: 31 May 2012

(C) The Author(s) 2012. This article is published with open access at Springerlink.com

\begin{abstract}
Orthogonal frequency and code division multiplexing (OFCDM) which can be extended orthogonal frequency and code division multiple access (OFCDMA) has a good performance, because it can mitigate multi path interference while maintaining high data rate. Furthermore, cooperative OFCDM relaying system provides these advantages, and this system can effectively use the limited resource with respect to data rate and each terminal's energy efficiency. Therefore we proposed cooperative OFCDMA relaying uplink system because of the user's requirement of the high data rate at uplink. However, a throughput performance decreases when this system employs many relays in case of each relay allocated code channel to separate each relay's signal. Therefore, in this paper, we present that the proposed system combines cyclic delay diversity due to complement throughput performance problem. DF (Decode-and-Forward) is assumed in this paper. It is considered that the users which want to send data to BS only decode the data from other users. In addition to that, we propose the adaptive cooperative communication for the OFCDMA uplink system. The proposed adaptive system can easily select the cooperative relay.
\end{abstract}

Keywords OFCDMA · CDD $\cdot$ Cooperative relaying $\cdot$ Relaying selection

\section{Introduction}

Future wireless communication systems increasingly need high reliability and throughput, because consumers require high quality of service (QoS). Specially, these conditions are necessary for uplink according to users desire that they quickly share the huge data. Orthogonal

J.-I. Baik · H.-K. Song $(\bowtie)$

412, uT communication institute, Choongmu-gwan, Sejong university,

98 Gunja-dong, Gwangjin-gu, Seoul 143-747, Korea

e-mail: songhk@sejong.ac.kr

J.-I. Baik

e-mail: bji0309@sdc.sejong.ac.kr 


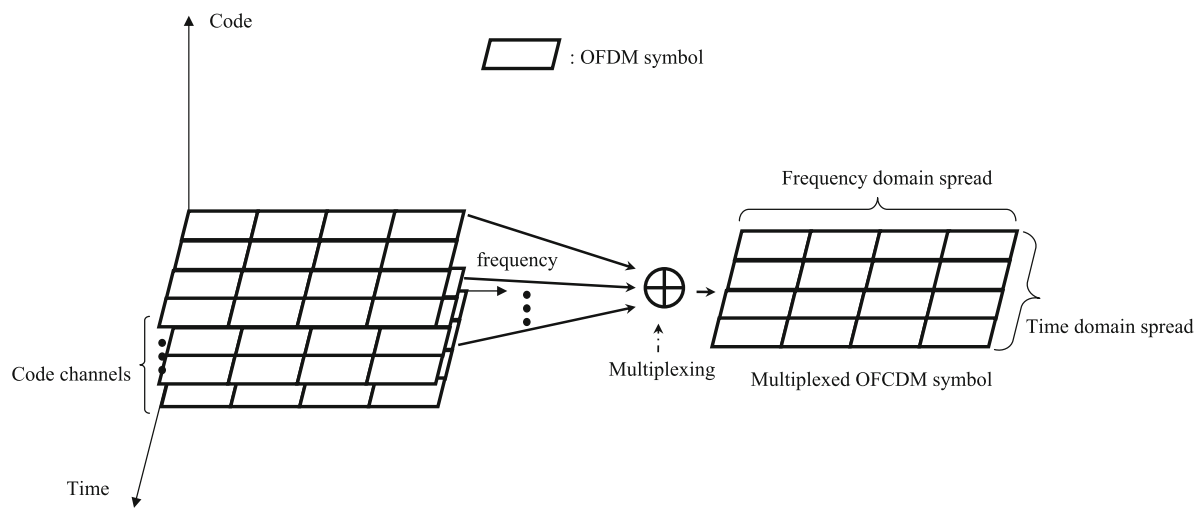

Fig. 1 The structure of the OFCDM symbol

frequency and code division multiplexing (OFCDM) is proposed to satisfy these conditions at downlink through diversity gain and the advantages of OFDM [1,2]

In the OFCDM, diversity gain can be achieved by frequency domain despreading owing to subcarriers which experienced the different fading [1-5]. To help understanding OFCDM, Fig. 1. shows the structure of an OFCDM symbol which explains the two-dimensional (2-D) spreading codes [3-5]. An OFCDM symbol combines OFDM and 2-D spreading codes, which are frequency domain and time domain as Fig.1. However OFCDM has been mostly studied for downlink system [1-6]. For these reason, in this paper, we extend OFCDM to orthogonal frequency and code division multiple access (OFCDMA) for efficiently embodying uplink system, and supporting multiple users as OFDMA [6]. In addition to that, it is chosen, because of the reason that OFCDMA transmits data more efficiently through allocating subcarriers and multi code in accordance with the channel condition of each user and its rate requirements and power.

For more efficient uplink transmission, we also consider cooperative OFCDMA relaying scheme shown. Cooperative OFCDM relaying scheme is proposed in [7]. This system provides high reliability through spatial diversity and frequency diversity. Moreover, this scheme effectively uses the limited resource with respect to data rate and each terminal's energy efficiency. This system provides high data rate, being able to enlarge coverage and small size, also.

Though cooperative OFCDMA relaying system has advantages as explained above, when many relays cooperate with the source for transmitting signal to the destination, throughput performance diminishes because of allocating the code channels at each relay in this system. Moreover, OFCDMA is hard to apply many frequency spreading codes when compared with OFCDM because of the restricted subcarriers. This problem leads to a decrease in the frequency diversity. Therefore we propose a system that combines cyclic delay diversity (CDD) and cooperative relaying system based on OFCDMA, and we call this system 'combined CDD cooperative OFCDMA relaying system'. CDD complements these restrictions through using all available code channels at each relay and spatial diversity gain without loss of the throughput even in the case direct-path condition is not good. CDD is a scheme that transmits cyclically delayed signals through different antennas $[8,9]$. Because, it causes that channel is made more frequency selective channel, CDD can get the spatial diversity when channel encoding is adopted [10-13]. And CDD has researched because of simple method [14]. So, the proposed system does not need extra highly complex processes at modulation and 
demodulation in comparison with cooperative OFCDMA relaying system. Therefore the proposed system can be expected to reduce time the implement of the high speed uplink system for the future consumer.

The rest of the paper is organized as follows. Section 2 describes conventional OFCDM system, the proposed combined CDD cooperative OFCDMA relaying system, and we also present the relaying selection scheme. In Sect. 3, we provide simulation results. We present the comparison of the proposed cooperative system and other systems. Finally, conclusion is given in Sect. 4.

\section{System Model}

In this paper, we consider only one cell system. However, this system can be extended multi cell environment. If we suppose multi cell system, we can assume that the same frequency is used in all the cells. The cellular system consists of a base-station per each cell. In this paper, all users do not overlap the whole subcarriers. To better describe the proposed system, the one cell is shown in Fig. 3. We will present the proposed system in sub-section in detail.

\subsection{Conventional OFCDM}

The simple transmitter and receiver are shown in Fig. 2 [2]. The process of creating OFCDM signal is as follows: First, information bit stream is converted serial to parallel. Next, converted multiple data streams are channel coded and modulated, respectively. And each modulated signal is spread by $2-\mathrm{D}$ spread codes. These codes go through the following

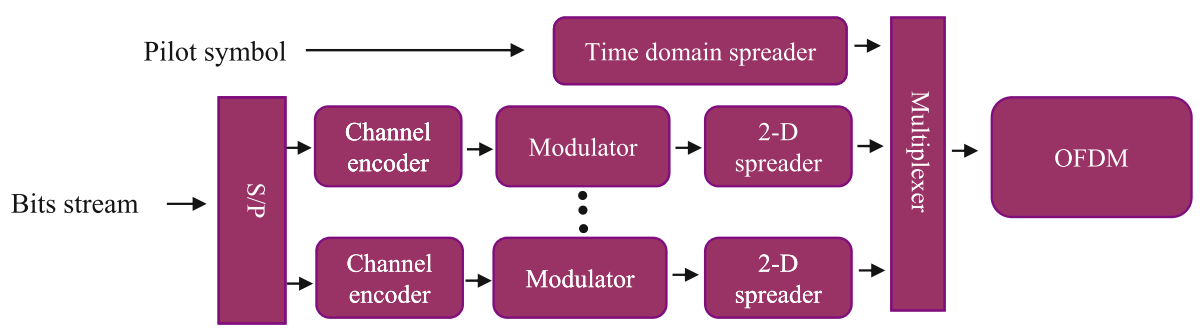

(a) Transmitter

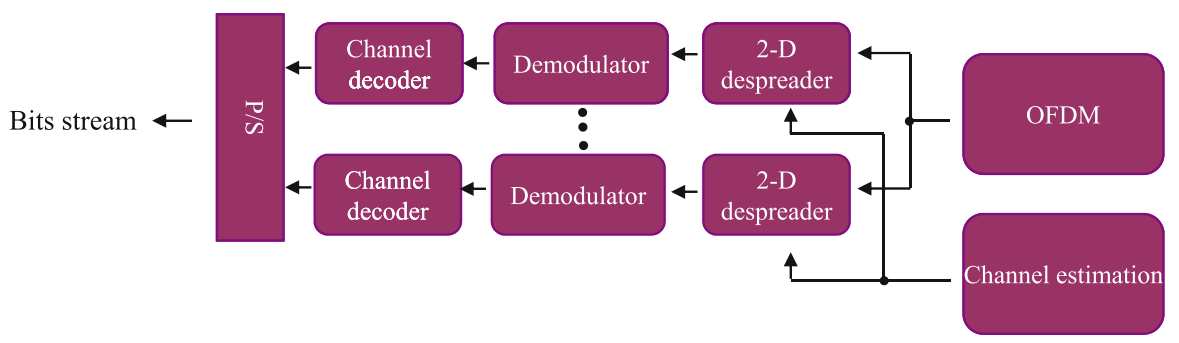

(b) Receiver

Fig. 2 Block diagram of OFCDM: a transmitter, b receiver 


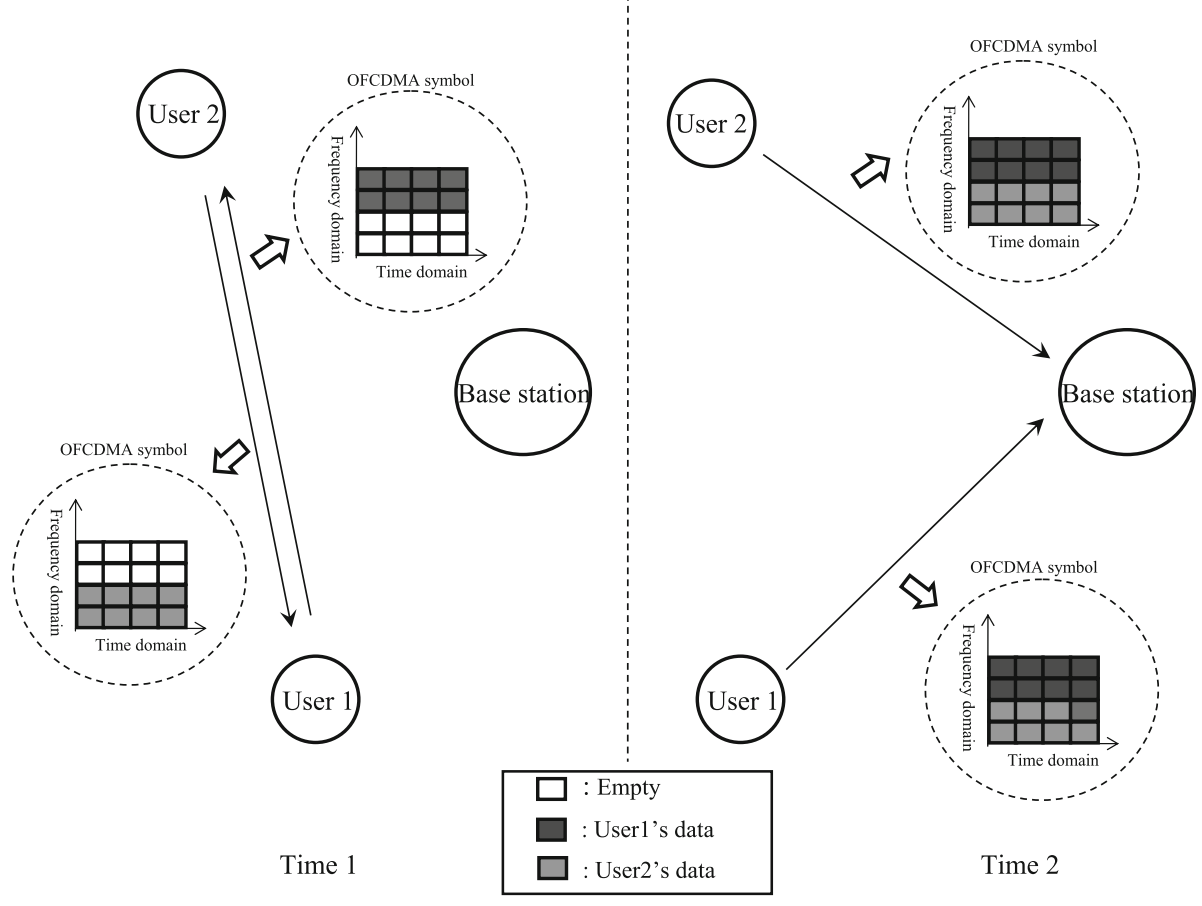

Fig. 3 An example of the combined CDD cooperative OFCDMA relaying system

process. The first processing is time spreading, and the second processing is frequency spreading. The 2-D spreading code can employ many other orthogonal code channels. For example, one time spreading code is $1,1,-1,-1$ and frequency spreading code is $1,-1$. We can design other time and frequency codes such as $-1,-1,1,1$ and $-1,1$, respectively. So, the total numbers of the spreading codes have to be less than eight code channel, in this case, because of maintaining the orthogonality. That is possible to keep data rate and high reliability. After finishing above process, all code channels are combined at code multiplexer. Then combined code passes IFFT and guard interval insertion blocks as OFDM system. So we can show OFCDM data symbol as

$$
S_{m, n}(t)=\sum_{k=1}^{K} C_{T, i}^{k} C_{F, j}^{k} x_{m, n, k}^{l} e^{j 2 \pi f_{n}(t-m T)}
$$

where, $C_{T}$ and $C_{F}$ are the time spreading code and the frequency spreading code, respectively. $k$ indicates $k$ th channel code, and $K$ means the total number of code channels. $x^{l}$ is $l$ th data stream. $S_{m, n}$ means $m$ th OFCDM symbol on $n$th subcarrier. In addition to that, the pilot signal can be expressed as

$$
P_{m, n}(t)=C_{T, i}^{r} p_{m, n, k} e^{j 2 \pi f_{n}(t-m T)}
$$

where, $C_{T}$ means a time spreading code, and $r$ indicates channel code index for the pilot, therefore the following result have to be satisfied as, $C_{T}^{r} \in C_{T}^{K} \cdot p_{m, n, k}$ is the pilot symbol. If the transmitter finishes these steps, it sends $S_{m, n}(t)+P_{m, n}(t)$ which is the multiplexed signal 
to the receiver. We suppose that the fading of channel is constant for the duration of the one symbol repetition, in time domain. Therefore received signal is represented as follows:

$$
\mathrm{r}_{\mathrm{m}, \mathrm{n}}(t)=\mathrm{H}_{\mathrm{m}, \mathrm{n}}(t)\left[S_{m, n}(t)+P_{m, n}(t)\right]+N_{m, n}(t)
$$

where $H_{m, n}$ is the channel. $N_{m, n}$ indicates a complex additive white Gaussian noise (AWGN) on $n$th subcarrier. Now, we explain the OFCDM demodulation. The first step of demodulation is the removal of the guard interval. And then, these signals are passed by FFT processing. After FFT and time domain despreading processing, pilot signal is used to channel estimate. On the other hands, other data code channels are passed by frequency domain despreader. The time domain despreading can be shown as:

$$
S_{T, m, n}=\sum_{i=1}^{I} C_{T, i}^{k} r_{m, n}
$$

and frequency domain despreading is

$$
S_{F, m, n}=\sum_{j=1}^{J} C_{F, j}^{k} S_{T, m, n}\left(\hat{H}_{m, n}\right)^{*}
$$

where $S_{T, m, n}$ and $S_{F, m, n}$ are the signals that finished the time and frequency domain despreads, respectively. $r_{m, n}$ means signal that is passed by FFT processing. $I$ is the total number of time spreading codes. And, $J$ denotes the number of frequency domain spreading codes. $\hat{H}_{m, n}$ means estimated channel. $(\cdot)^{*}$ is the complex conjugate of $(\cdot)$. If the despreading is finished, modulation and channel decoder are performed.

\subsection{Proposed CDD Combined Cooperative OFCDMA Relaying System}

Figure 3 shows the example of this system. For simple interpreting, a half subcarriers of the whole subcarriers at this system are allocated for each user, and only one code channel is used in this figure. This system consists of two time intervals. First time interval, each user sends data to the other user. First time signal can be represented as

$$
\begin{aligned}
S_{m, u}^{U 1}(t) & =\sum_{k=1}^{K} C_{T, i}^{k} C_{F, j}^{k} x_{m, u, k}^{U 1, l} e^{j 2 \pi f_{u}(t-m T)}, \\
S_{m, u}^{U 2}(t) & =\sum_{k=1}^{K} C_{T, i}^{k} C_{F, j}^{k} x_{m, u, k}^{U 2, l} e^{j 2 \pi f_{u}(t-m T)} .
\end{aligned}
$$

Equations (6) and (7) mean the user1's transmitted signal and the user2's transmitted signal, respectively. U1 means user 1; U2 denotes user 2. $u$ represents the index that is assigned subcarriers according to the user. Other indexes are the same as Eq. (1). Each user decodes the received data as described in previous subsection. If finishing the decoding, each user encodes again with the partner's data and users own data. This signal can be written as

$$
\begin{aligned}
& S_{m, n}^{B S 1}(t)=\sum_{k=1}^{K} C_{T, i}^{k} C_{F, j}^{k} d_{m, n, k}^{l} e^{j 2 \pi f_{n}(t-m T)}, \\
& S_{m, n}^{B S 2}(t)=\sum_{k=1}^{K} C_{T, i}^{k} C_{F, j}^{k} d_{m, n, k}^{l} e^{j 2 \pi f_{n}(t-m T)} \delta
\end{aligned}
$$


where $B S 1$ and $B S 2$ means that $\mathrm{U} 1$ or $\mathrm{U} 2$ sends the signal to base station (BS), respectively. $d_{m, n, k}^{l}$ represents $l$ th data stream; we assume that $d_{m, n, k}^{l}$ is the signal after channel coding with interleaving. $m$ is OFCDMA symbol index and $n$ means $n$th subcarrier. Finally, $\delta$ is the cyclic delay term that is $e^{-2 \pi \delta_{c y c} n / N} . N$ is the total number of subcarriers and $\delta_{c y c}$ is a matter of the performance of CDD. The optimum cyclic delay was presented in [9]. After re-encoding, $\mathrm{U} 1$ and $\mathrm{U} 2$ transmit these signals to the BS, over the second time interval. Therefore, BS receives

$$
r_{m, n}(t)=H_{m, n}^{U 1 B}(t) S_{m, n}^{B S 1}(t)+H_{m, n}^{U 2 B} S_{m, n}^{B S 2}(t)+N_{m, n}(t)
$$

where $H_{m, n}^{U 1 B}$ represents the channel between the $\mathrm{U} 1$ and BS, and $H_{m, n}^{U 2 B}$ represents the channel between the U2 and BS. For simple explanation, pilot is omitted in this equation. Hence, BS decodes this signal as follows:

$$
\begin{aligned}
S_{T, m, n} & =\sum_{i=1}^{I} C_{T, i}^{k} r_{m, n}, \\
S_{F, m, n} & =\sum_{j=1}^{J} C_{F, j}^{k} S_{T, m, n}\left(\hat{H}_{m, n}^{e}\right)^{*}
\end{aligned}
$$

where Eqs. (10) and (11) represent the time domain despreading and frequency domain despreading, respectively. Other indexes is the same as Eqs. (4), and (5). $H_{m, n}^{e}$ is the practical transfer function of the signal $S_{m, n}^{B S 1}$ and $S_{m, n}^{B S 2}$ as below:

$$
\hat{H}_{m, n}^{e}=\frac{1}{\sqrt{2}}\left(H_{m, n}^{U 1 B}+H_{m, n}^{U 2 B} e^{-j 2 \pi n \delta_{c y c} / N}\right) .
$$

The system that bases on OFCDMA is difficult to use many frequency spreading codes because of the limitation of the subcarriers. That causes a decrease of the diversity gain.

\subsection{The Adaptive Cooperative Relaying Scheme for the Proposed System}

In cooperative communication, the relaying selection is the important problem. If the node in bad channel condition is selected as partner, the performance can be worse than non-cooperative communication system. Therefore in this section, we present the adaptive cooperative transmission for the OFCDMA uplink system. The detailed process of proposed system is as follows. First, all nodes add the Cyclic Redundancy Check (CRC) after data stream, and then users which need to transmit to the BS send data to other users (candidates). In the first time slot, transmit signal is the same as Eq. (6). If candidates want to send data and to be relay node, they decode this signal and check the CRC. In the other case they do not decode the data. For example, if the proposed system employs $4 \times 2$ spreading code, the candidates receive the 8 data streams (maximum value). In this case, the various situations can be considered. The candidates calculate the total number of the successful check, and then this information is transmitted by candidates to BS. The best situation is when the number of errors is ' 0 ', and the worst situation is when the number of errors is ' 8 '. In this paper, because this information is important, it is considered that CRC checking information is added at the header signal. The BS checks the number of errors in the data streams, and decides the best relay. If some of candidates have the fewest number of CRC check error compared with other candidates, BS requests SNR check message to them, and then they compute the SNR. After this process, they transmit the information of SNR to the BS. BS also uses this information to decide the 
best relay by the necessity. Finally, users and the partners transmit the data to BS. In the proposed system, candidates which have bad SNR do not need to estimate SNR. Therefore, this process can decrease complexity to users.

\section{Simulation Result}

In this section, we provide performance results for the proposed system. As a channel model, Rayleigh fading channel where each path is independently faded is adopted. It is assumed that the channel attenuation and phase shift are essentially fixed for the duration of one signaling block. It is also supposed that the channel state information (CSI) is perfectly known. We limit our study to the one-cell system, in this paper. All simulations are supposed that uplink FFT size is 1024; inter-user FFT size is 512. We apply a convolutional code with code rate of $1 / 2$. QPSK is chosen for symbol mapping. Figures 4 , and 5 . show the bit error rate (BER) performance. Here, we define a term of "SNR gap" which means the difference of SNR between user-to-BS channel and inter-user channel. Figure 4. represents the performance when inter-user channel is $5 \mathrm{~dB}$ better than user-to-BS channel. Figure 4 . describes the performance of non cooperative OFCDMA system, combined CDD cooperative OFCDMA system (CC cooperative OFCDMA system), allocated code cooperative OFCDMA system (AC cooperative OFCDMA system), cooperative OFDMA system, and $2 \times 1$ OFCDMA system. AC cooperative OFCDM system is studied in [1]. This system allocates different code channels to each user. AC cooperative OFCDMA system gives better BER performance than the proposed system in Fig. 4. When CC cooperative OFCDMA system and

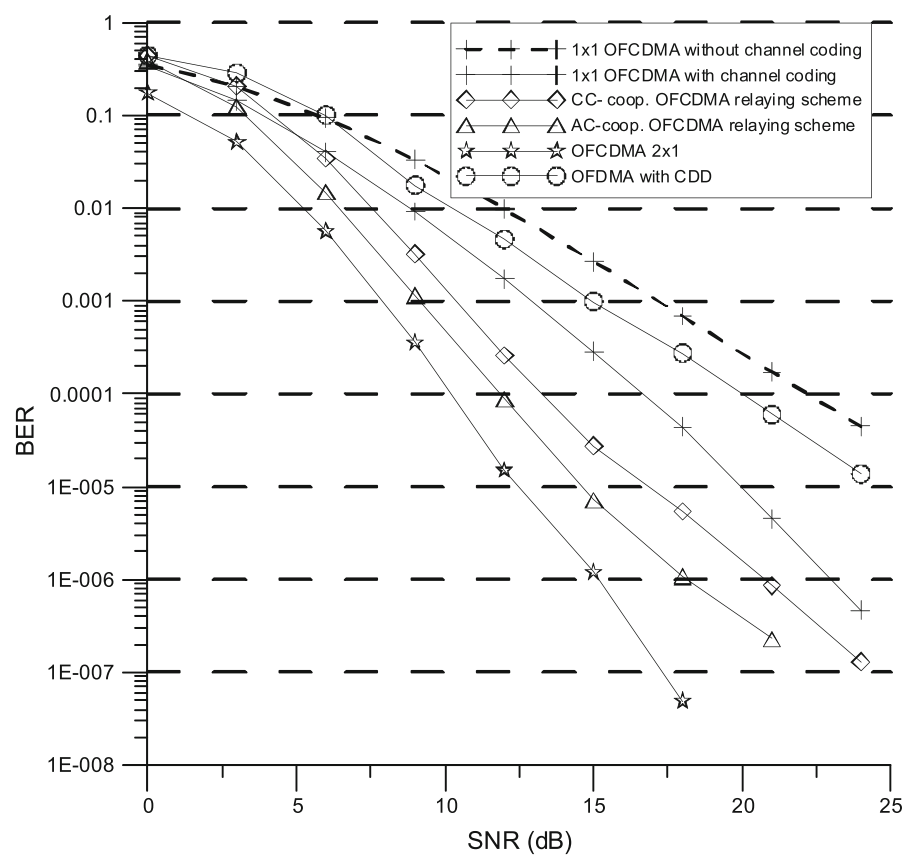

Fig. 4 BER performance comparison of noncooperative OFCDMA, OFDMA cooperative, combined CDD cooperative OFCDMA, allocated code cooperative OFCDMA systems in accordance with the SNR gap of $5 \mathrm{~dB}$, and $2 \times 1$ OFCDMA system 


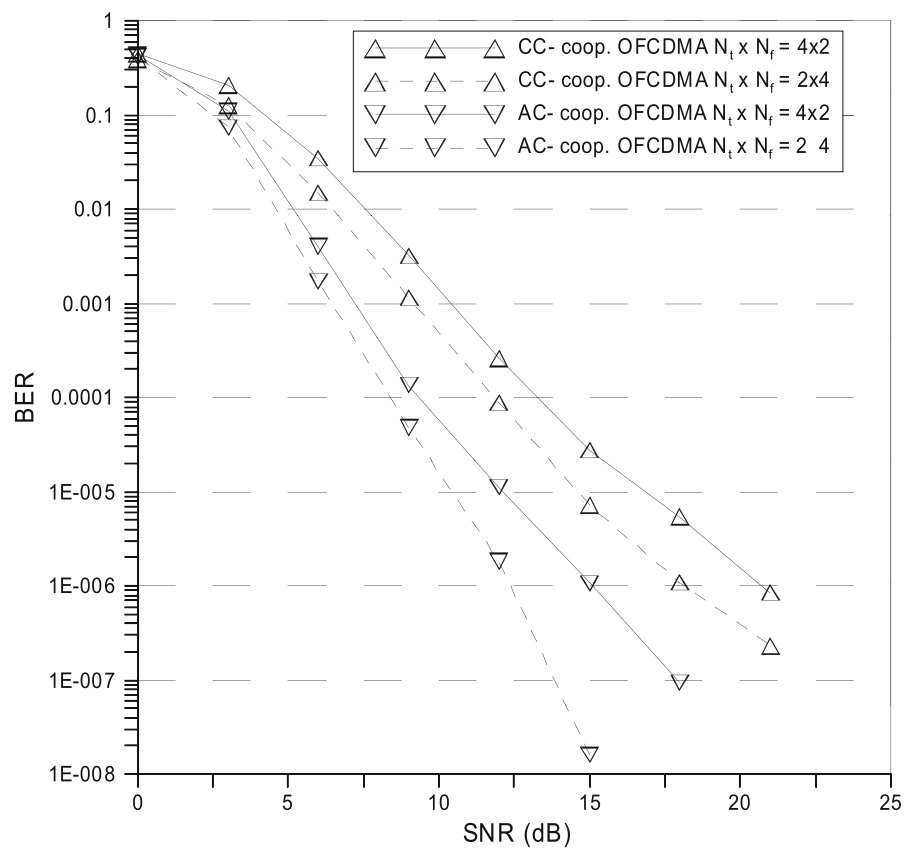

Fig. 5 BER performance comparison of combined CDD cooperative OFCDMA in accordance with different code channel design

AC OFCDMA cooperative system compares with conventional $1 \times 1$ OFCDMA system, we find that cooperative system has good SNR performance about 3 and $4 \mathrm{~dB}$ at $10^{-4}$, respectively. Also, because partner node has error when it decodes data, $2 \times 1$ OFCDMA system gives better BER performance than AC OFCDMA cooperative system and CC OFCDMA cooperative system. Lastly, the BER performance of proposed OFCDMA system is better than cooperative transmission scheme based on OFDMA system because of the frequency diversity gain by frequency spreading codes. Figure 5. shows the BER performance of CC cooperative OFCDMA system and AC cooperative OFCDMA system in accordance with different code channel design. $N_{t}$ means the total number of time spreading codes, and $N_{f}$ means the number of frequency spreading codes. We find that $N_{f}$ is more important than $\mathrm{Nt}$ to get the diversity. In other words, it can be said that code design should be considered in accordance with channel condition. In this simulation, $N_{t}$ can be applied to control data rate.

The BER performance comparison of adaptive CC cooperative OFCDMA system, adaptive AC cooperative OFCDMA system, and fixed cooperative relaying system is showed in Fig. 6. The adaptive CC and AC cooperative OFCDMA system applies the CRC relaying selection technique. The cooperative OFCDMA which adopts the proposed relaying selection is supposed that this system consists of 6 users, and SNR gap is $-5,-2,0,2,3$, and $5 \mathrm{~dB}$. The fixed AC and CC cooperative OFCDMA is made up of only one relay ( $\mathrm{SNR}$ gap $=5 \mathrm{~dB}$ ). We can find the fact that fixed OFCDMA cooperative system that SNR gap is $5 \mathrm{~dB}$ gives almost same performance as the proposed cooperative system. It means that user which has $5 \mathrm{~dB}$ is almost selected. In other words, this selection technique can find the partner which has the best channel state. In this simulation, only one user is considered to be a partner. However, if many users are selected as partner, AC cooperative system decreases the performance 


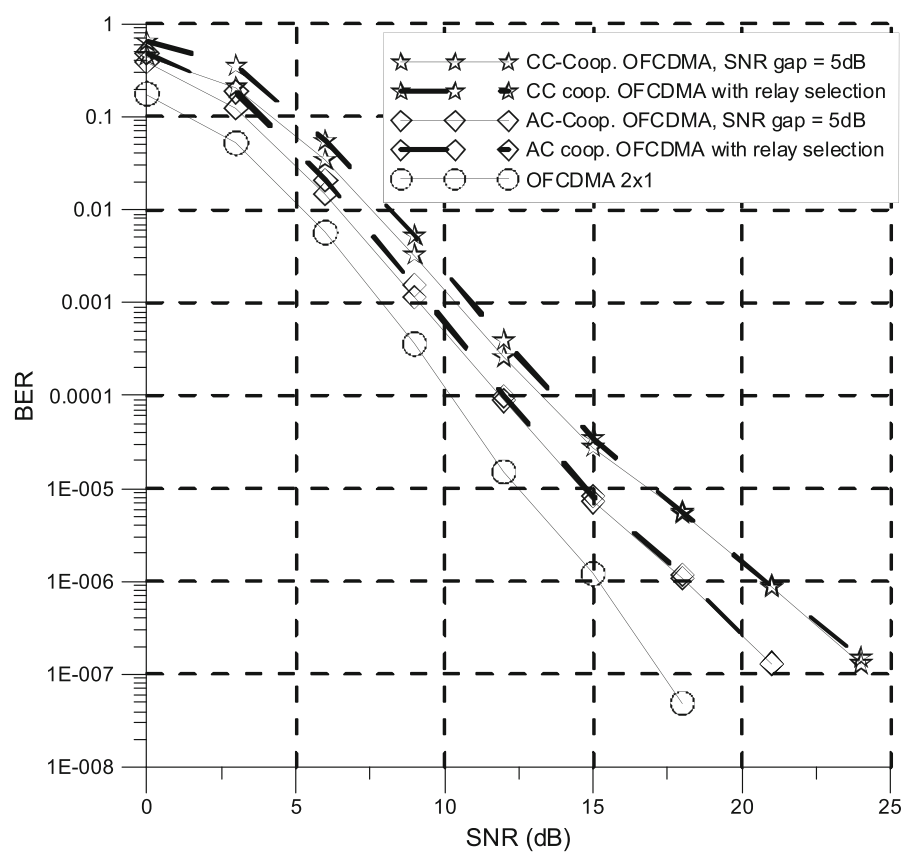

Fig. 6 BER performance comparison of adaptive CC OFCDMA cooperative system, adaptive AC OFCDMA cooperative system, and fixed OFCDMA cooperative system with SNR gap of $5 \mathrm{~dB}$

because of the limit of the total number of CRC information when this system compares with CC OFCDMA cooperative system.

Figure 7. depicts the throughput performance of $\mathrm{CC}$ cooperative OFCDMA system and AC cooperative OFCDMA system. In this figure, we suppose that multi-code channel interference (MCI) do not exists. In this case, this figure is shown that two user cooperate each other. And, we can find that CC cooperative OFCDMA system is given better throughput performance than AC cooperative OFCDMA system. Specially, if AC cooperative OFCDMA system has a lot of users, this system requires more code channels. That causes the serious decrease of throughput performance. On the other hand, because the proposed system is possible to use all code channels at each node, the proposed system is not diminished throughput performance by code channels. So this result represents that the proposed system is suitable for the system which employs many relays.

Figure 8. represents BER performance of CC cooperative OFCDMA system according to cyclic delay value. In this simulation, we fixed SNR at $18 \mathrm{~dB}$. CC cooperative OFCDMA system has the best BER performance when cyclic delay value is $1 / 4$ or $3 / 4$ as in OFDM system. However, this result is different from OFDM system with respect to having more similar the best BER performance and bad BER performance at other cyclic delay values in CC cooperative OFCDMA system. Finally, the combined channel is shown in Fig. 9. We only show the 1-100 subcarriers. This figure can find that combined channel frequency response provides much more frequency selective fading than U1 to BS and U2 to BS channel. Therefore CC cooperative OFCDMA system can obtain spatial diversity. 


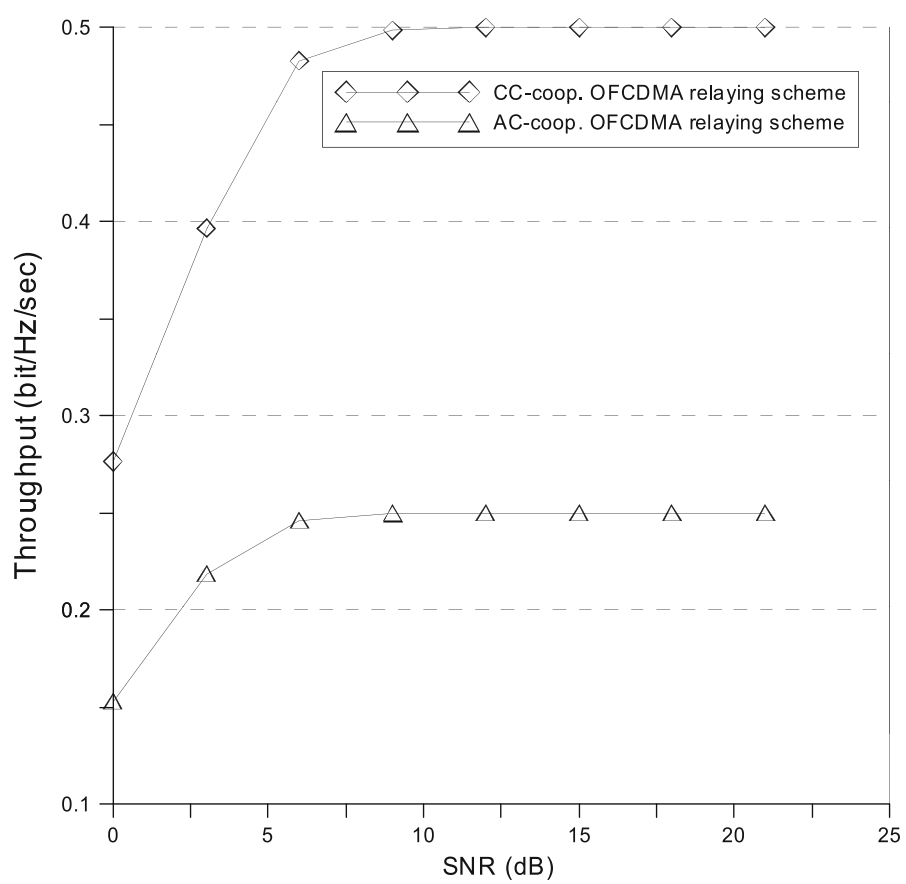

Fig. 7 Throughput performance comparison of combined CDD cooperative OFCDMA, and allocated code cooperative OFCDMA systems in accordance with the SNR gap of $5 \mathrm{~dB}$

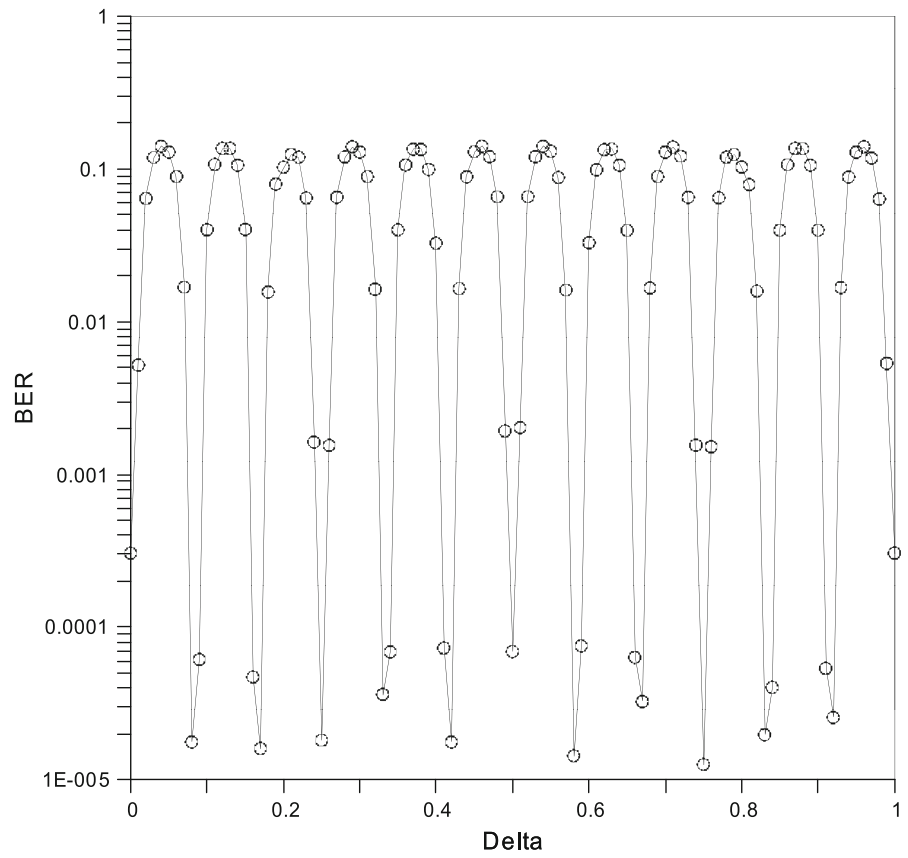

Fig. 8 BER performance of combined CDD cooperative OFCDMA in accordance with the different cyclic delay values at SNR $18 \mathrm{~dB}$ 


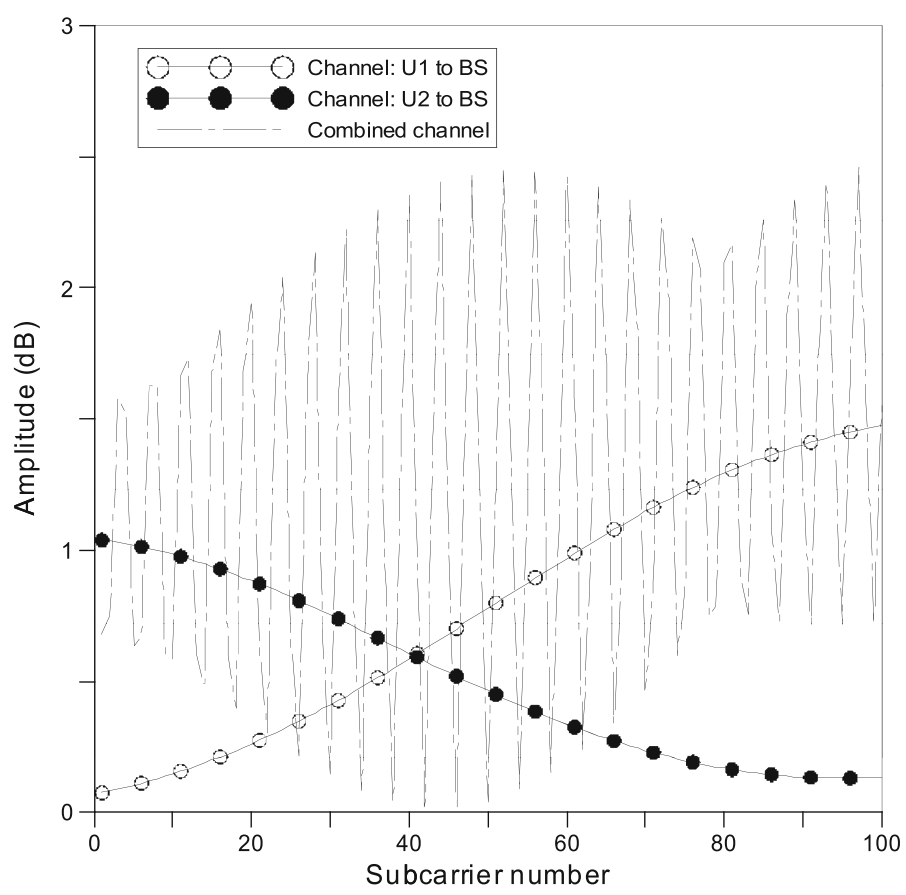

Fig. 9 An example of channel frequency responses at combined CDD cooperative OFCDMA

\section{Conclusion}

In a new cooperative relaying system which combines CDD based on OFCDMA has been presented in this paper. We have shown that the proposed system can obtain both frequency diversity and spatial diversity. For this reason, the proposed system can effectively get more diversity gain than simple OFCDMA system. We also find that AC cooperative OFCDMA system which allocates different code channel to each user provides better BER performance than the proposed cooperative OFCDMA system. However, because the proposed cooperative OFCDMA system can use more code channels than AC cooperative OFCDMA system, the proposed cooperative system provides better throughput performance than AC cooperative OFCDMA system. We present the adaptive relaying technique which has low complexity. The proposed relaying selection technique is shown that this technique can find the partner which has the best SNR.

Acknowledgments This research was supported by the MKE(The Ministry of Knowledge Economy), Korea, under the Convergence-ITRC(Convergence Information Technology Research Center) support program (NIPA-2012-H0401-12-1003) supervised by the NIPA(National IT Industry Promotion Agency) and this research was supported by the MKE(Ministry of Knowledge Economy), Korea, under the ITRC(Information Technology Research Center) support program supervised by the NIPA(National IT Industry Promotion Agency) (NIPA-2012-H0301-12-2007).

Open Access This article is distributed under the terms of the Creative Commons Attribution License which permits any use, distribution, and reproduction in any medium, provided the original author(s) and the source are credited. 


\section{References}

1. Zhou, Y., Wang, J., \& Sawahashi, M. (2005). Downlink transmission of broadband OFCDM systems-part I: Hybrid detection. IEEE Transactions on Communications, 53(4), 718-729.

2. Zhou, Y., Ng, T. -S., Wang, J., Higuchi, K., \& Sawahashi, M. (2006). Downlink transmission of broadband OFCDM systems-part IV: Soft decision. IEEE Transactions on Communications, 24(6), 1208-1220.

3. Zhou, Y., Wang, J., \& Sawahashi, M. (2007). Two dimensionally spread OFCDM systems for 4G mobile communications. In Proceedings of IEEE. TENCON (pp. 1-4).

4. Zhou, Y., Ng, T. -S., Wang, J., Higuchi, K., \& Sawahashi, M. (2008). OFCDM: A promising broadband wireless technique. IEEE Communications Magazine, 46(2), 39-49.

5. Zhou, Y., Wang, J., \& Sawahashi, M. (2007). Two dimensionally spread OFCDM systems for 4G mobile communications. In Proceedings of IEEE. TENCON (pp. 1-4).

6. Caldwell, R., \& Anpalagan, A. (2006). Performance analysis of subcarrier allocation in two dimensionally spread OFCDM systems. In Proceedings of IEEE. VTC (pp. 1-5).

7. Baik, J. -I., Kim, J. -H., \& Song, H. -K. (2008). Standard conformable antenna diversity techniques for OFDM and its application to the DVB-T system. In Proceedings of ATNAC (pp. 134-137).

8. Dammann, A., \& Kaiser, S. (2001). Performance improvement of cooperative relaying scheme based on OFCDM in UWB channel. In Proceedings of IEEE Globecom, Vol. 5. (pp. 3100-3105)

9. Bauch, G., \& Malik, J. S. (2006). Cyclic delay diversity with bit-interleaved coded modulation in orthogonal frequency division multiple access. IEEE Transactions on Wireless Communications, 5(8), 20922100.

10. Wei, C., Hu, T., \& You, X. (2006). Cyclic delay diversity performance in OFDMA based system. In Proceedings of IEEE MILCOM (pp. 1-7).

11. Plass, S., Dammann, A., \& Sand, S. (2008). An Overview of cyclic delay diversity and its applications. In Proceedings of IEEE VTC (pp. 1-4).

12. Zhang, F., Zhang, Y., Malik, W. Q., Allen, B., \& Edwards, D. J. (2008). Optimum receive antenna selection for transmit cyclic delay diversity. In Proceedings of IEEE ICC (pp. 3829-3833).

13. Chen, H. Z. B., Schober, R., Lampe, L., \& Allen, B. (2008). Generalized Cyclic delay diversity for orthogonal frequency division multiplexing. In: Proceedings of IEEE VETECF (pp. 526-530).

14. Khan, F., \& Van Rensburg, C. (2006). An adaptive cyclic delay diversity technique for beyond 3G/4G wireless systems. In IEEE Proceedings of VTC (pp. 1-6).

\section{Author Biographies}

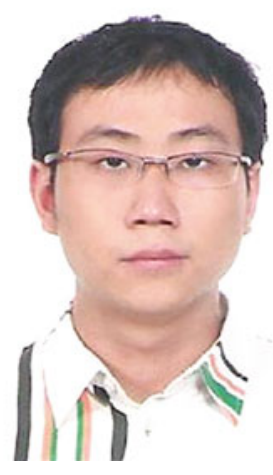

Jung-In Baik was born in Seoul, Korea, in 1984. He is currently with the Department of information and communication engineering, Sejong University, Korea. His research interests are Cooperative MIMO technique and Ultra wide band (UWB) system. 


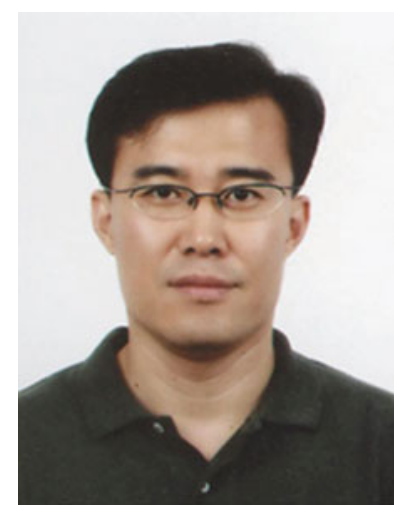

Hyoung-Kyu Song was born in CungCheong-Bukdo, Korea on May 14th in 1967. He received B.S., M.S., and Ph.D. degrees in electronic engineering from Yonsei University, Korea, in 1990, 1992, and 1996, respectively. From 1996 to 2000 he had been managerial engineer in Korea Electronics Technology Institute (KETI). Since 2000 he has been an associate professor of the Department of information \& communication engineering, Sejong University, Korea. His research interests include digital and data communications, information theory, and their applications with an emphasis on mobile communications. 